\title{
Dynamic probabilities of restrictions in state space models: An application to the Phillips curve
}

\author{
Gary Koop \\ Department of Economics \\ University of Strathclyde \\ Scotland \\ Gary.Koop@strath.ac.uk \\ Roberto Leon-Gonzalez \\ National Graduate Institute for Policy Studies \\ Japan \\ rlg@grips.ac.jp \\ Rodney W. Strachan \\ School of Economics \\ University of Queensland \\ Australia \\ r.strachan@uq.edu.au
}

December 2007

\begin{abstract}
Empirical macroeconomists are increasingly using models (e.g. regressions or Vector Autoregressions) where the parameters vary over time. State space methods are frequently used to specify the evolution of parameters in such models. In any application, there are typically restrictions on the parameters that a researcher might be interested in. This motivates the question of how to calculate the probability that a restriction holds at a point in time without assuming the restriction holds at all (or any other) points in time. This paper develops methods to answer this question. In particular, the principle of the Savage-Dickey density ratio is used to obtain the time-varying posterior probabilities of restrictions. We use our methods in a macroeconomic application involving the Phillips curve. Macroeconomists are interested in whether the long-run Phillips curve is vertical. This is a restriction for which we can calculate the posterior probability using our methods. Using U.S. data, the probability that this restriction holds tends to be fairly high, but decreases slightly over time (apart from a slight peak in the late 1970s). We also calculate the probability that another restriction, that the NAIRU is not identified, holds. The probability that it holds fluctuates over time with most evidence in favor of the restriction occurring after 1990.
\end{abstract}

Key Words: Bayesian, state space model, Savage-Dickey density ratio, time varying parameter model.

JEL Classification: C11, C32, E52 


\section{Introduction}

Many recent papers, particularly in the field of macroeconomics, have worked with extensions of regressions or Vector autoregressions (VARs) where the parameters can change over time. With a wide variety of data sets, there is strong empirical evidence that such time-varying parameter (TVP) models are necessary to capture data properties of economic relevance (e.g. the evolution of transmission mechanisms or the processes generating the exogenous shocks). The TVP-VARs of (among many others) Cogley and Sargent (2001, 2005), Koop, Leon-Gonzalez and Strachan (2007) and Primiceri (2005) have the form:

$$
\begin{aligned}
y_{t} & =Z_{t} \alpha_{t}+\varepsilon_{t} \\
\alpha_{t+1} & =\alpha_{t}+\eta_{t},
\end{aligned}
$$

where $y_{t}$ is a vector of dependent variables and $Z_{t}$ contains appropriate lags of the dependent variable and deterministic terms.

TVP-VARs are a popular and powerful tool in modern macroeconomic research, however they suffer from some drawbacks. Firstly, TVP-VARs are reduced form models which do not impose any restrictions on the coefficients. However, economic theory often suggests restrictions on $\alpha_{t}$ and, in applications involving traditional VARs (i.e. with parameters which are constant over time) such restrictions are often tested or imposed. Secondly, TVP-VARs can be over-parameterized, rendering it hard to obtain precise inference. Imposing restrictions on TVP-VARs can reduce such over-parameterization problems. These considerations suggest that developing methods for calculating the probability that restrictions on the coefficients hold in state space models such as the TVP-VAR is of interest. This is what we do in this paper using Bayesian methods. Since $\alpha_{t}$ varies over time, the probability of a restriction holding can also vary over time. This motivates our terminology "dynamic posterior probabilities" of a restriction holding.

In this paper, we develop methods for calculating such dynamic posterior probabilities for restrictions on state variables such as $\alpha_{t}$ using output from standard simulation algorithms for state space models (see, e.g., Durbin and Koopman, 2002) and the principle of the Savage-Dickey density ratio (SDDR), see Verdinelli and Wasserman (1995). Although our focus is on macroeconomic applications with time-varying parameter models, 
we stress that these methods are of more general use for calculating the probability of restrictions on states in any state space model.

There is a huge variety of restrictions that might be of interest in macroeconomics. In the empirical part of this paper, we consider an application to the Phillips curve and show how the underlying theory implies certain restrictions. Another example is King, Plosser, Stock and Watson (1991). This paper used the balanced growth hypothesis to motivate restrictions on the relationships between income, investment and consumption. This is an example where economic theory motivates restrictions. Another example is Lettau and Ludvigson (2004), which investigates the relationship among asset wealth, income and consumption. As discussed in that paper and further investigated in Koop, Potter and Strachan (2007), an empirically-motivated restriction (a weak exogeneity restriction) is important in obtaining a key result. These papers all use models where the parameters are constant over time and, thus, they can simply calculate the probability that a restriction holds using familiar statistical methods. However, to our knowledge there is little work which focusses on calculating the probability that restrictions hold in TVP models. Given this lack and the empirical importance of TVP models, it is natural that we consider this problem and develop methods of obtaining the posterior probability that a restriction holds at a particular point in time, without requiring the restriction to be imposed at any other time.

It is also important to stress what we are not doing. Firstly, we are not interested in calculating the Bayes factor comparing TVP models to comparable models with constant parameters (e.g. comparing a TVP-VAR to a VAR). We can do this using our methods, but the existing literature already provides good ways of doing this. For instance, the bridge sampling methods of Fruhwirth-Schnatter (2004) can be easily adapted to do this. Secondly, we are not developing methods for the recursive testing of a restriction. The dynamic posterior probabilities of restrictions we obtain are conditional upon the full sample and, in this sense, allow for more efficient inference. That is, if we write the restriction being tested as $A \alpha_{t}=\alpha^{*}$ for some known matrix $A$ and vector $\alpha^{*}$, we calculate $\operatorname{Pr}\left(A \alpha_{t}=\alpha^{*} \mid y\right)$ where $y=\left(y_{1}^{\prime}, . ., y_{T}^{\prime}\right)^{\prime}$ denotes the full sample. So we are computing the probability that the restriction holds at time $t$, but does not hold elsewhere, and this probability is conditional upon all information available. We are not calculating $\operatorname{Pr}\left(A \alpha_{t}=\alpha^{*} \mid y_{1}, . ., y_{t}\right)$ as would be done in a recursive approach (although, as noted below, it would be easy to modify our methods to calculate this latter probability). Thus, our methods address questions of the form: "What is the probability that a restriction/theory holds at 
time $t$, given all the information in our data set?".

The structure of the paper is as follows. In Section 2 we present the state space model and associated posterior simulation methods based on the Kalman filter. In Section 3 we present the basic ideas behind the Savage-Dickey density ratio and demonstrate how this, combined with posterior simulator output, can be used to calculate the dynamic posterior probabilities of any linear restriction on the states. Section 4 discusses two extensions to our basic approach. These are the calculation of dynamic posterior probabilities of restrictions which involve states in different time periods (e.g. restrictions like $\alpha_{t+1}=\alpha_{t}$ ) and which involve nonlinear restrictions. The Section 5 uses these methods in an application relating to the Phillips curve. Section 6 concludes.

\section{Basic Posterior Results for the State Space Model}

We begin by defining our model (which is slightly more general than the one discussed in the introduction). This is a standard state space model, although we focus on the TVP interpretation of it. Let $y_{t}$ for $t=1, . ., T$ denote a vector of observations on $p$ dependent variables and $y=\left(y_{1}^{\prime}, y_{2}^{\prime}, \ldots, y_{T}^{\prime}\right)^{\prime}$ be the $T p \times 1$ vector of all the observations on all dependent variables. The measurement and state equations in the state space model are given by

$$
\begin{aligned}
y_{t} & =Z_{t} \alpha_{t}+\varepsilon_{t}, \quad \varepsilon_{t} \sim N\left(0, H_{t}\right) \\
\alpha_{t+1} & =T_{t} \alpha_{t}+\eta_{t} \quad \eta_{t} \sim N\left(0, Q_{t}\right), t=1, \ldots, T, \text { and } \\
\alpha_{1} & \sim N\left(a_{1}, P_{1}\right) .
\end{aligned}
$$

We further assume $\varepsilon_{t}$ and $\eta_{s}$ are independent for all $t$ and $s$. The vector $Z_{t}$ is of dimension $1 \times m$ such that $\alpha_{t}$ is a $m \times 1$ vector, $T_{t}$ is an $m \times m$ matrix and $\eta_{t}$ is an $n \times 1$ vector.

Many popular macroeconomic models fit in this framework. A TVP regression model (such as we use in our empirical section), has $p=1$ and $Z_{t}$ containing observations on explanatory variables and their lags and lagged dependent variables. The TVP-VARs of Cogley and Sargent (2005) and Primiceri (2005) have $T_{t}=I$ and $Z_{t}$ containing an intercept and suitable lags of the dependent variables and $H_{t}$ taking a particular multivariate 
stochastic volatility form. Cogley and Sargent (2001) adopts the same form, but restricts $H_{t}$ to be constant over time. Adding a term of the form $X_{t} \beta$ to (1) is a trivial extension which we will not consider to keep the notation uncluttered. It is also worth noting that, in most macroeconomic applications it is important to allow for stochastic volatility in $\varepsilon_{t}$. In our empirical application, we do allow for stochastic volatility. However, since our theoretical derivations relate to $\alpha_{t}$, we will simply leave $H_{t}$, the time-varying measurement error variance, unspecified at this stage.

Posterior simulation of state space models can be done using any one of several algorithms (e.g. in this paper we use the algorithm of Durbin and Koopman, 2002). This can be used as a block in a Markov Chain Monte Carlo (MCMC) algorithm for carrying out posterior simulation in the state space model. That is, conditional on $H_{t}, T_{t}, Q_{t}, a_{1}$ and $P_{1}$, the algorithm of Durbin and Koopman (2002) can be used to draw $\alpha_{t}$ for $t=1, . ., T$. Conditional on $\alpha_{t}$, posterior draws of $H_{t}, T_{t}, Q_{t}, a_{1}$ and $P_{1}$ can be taken (although often some of these are set to pre-selected values such as $T_{t}=I$ ). Given conditionally conjugate priors, the formulae for the posterior draws of $H_{t}, T_{t}, Q_{t}, a_{1}$ and $P_{1}$ are standard (see, e.g., Koop, 2003, pages 196-197) so we will not discuss them here (see the appendix for details) and will not explicitly list these conditioning arguments in our subsequent discussion of the conditional posterior of $\alpha_{t}$.

For this model, the posterior distribution (conditional on of $H_{t}, T_{t}, Q_{t}, a_{1}$ and $P_{1}$ ) of the vector $\alpha=$ $\left(\alpha_{1}^{\prime}, . ., \alpha_{T}^{\prime}\right)^{\prime}$ is Normal and can be pinned down by its mean and covariance matrix. Standard Kalman filtering and smoothing methods can be used to obtain these (see, e.g., Durbin and Koopman, 2001, chapter 4). To fix notation, we describe the relevant steps of the filter and smoother here.

The Kalman filter is a sequence of recursive operations running from $t=1, . ., T$ that simplify and speed algorithms for drawing from $\alpha_{t}$. The Kalman filter provides $E\left(\alpha_{t} \mid y_{1}, . ., y_{t}\right)=a_{t}$ and the variance $\operatorname{var}\left(\alpha_{t} \mid y_{1}, . ., y_{t}\right)=$ $P_{t}$ by evaluating:

$$
\begin{array}{rlr}
v_{t} & =y_{t}-Z_{t} a_{t} & F_{t}=Z_{t} P_{t} Z_{t}^{\prime}+H_{t} \\
K_{t} & =T_{t} P_{t} Z_{t}^{\prime} F_{t}^{-1} & L_{t}=T_{t}-K_{t} Z_{t} \\
a_{t+1} & =T_{t} a_{t}+K_{t} v_{t} & P_{t+1}=T_{t} P_{t} L_{t}^{\prime}+Q_{t} .
\end{array}
$$

As a digression, in the introduction, we distinguished our question of interest: "What is the probability that 
a restriction/theory holds at time $t$, given all the information in our data set?" from one that could be answered

by recursive estimation techniques ("What is the probability that a restriction/theory holds at time $t$, given the information available at time $t$ ?"). If one were interested in the latter question, we could use $E\left(\alpha_{t} \mid y_{1}, . ., y_{t}\right)$ and $\operatorname{var}\left(\alpha_{t} \mid y_{1}, . ., y_{t}\right)$ along with the SDDR (described in the next section) to obtain recursive dynamic posterior probabilities of restrictions. That is, we could compute $\operatorname{Pr}\left(A \alpha_{t}=\alpha^{*} \mid y_{1}, . ., y_{t}\right)$ using Kalman filter output and the SDDR.

Our objective, however, is to compute the posterior probability that the restrictions hold at time $t$ given the full sample: $\operatorname{Pr}\left(A \alpha_{t}=\alpha^{*} \mid y\right)$. For this we require knowledge of $\widehat{\alpha}_{t}=E\left(\alpha_{t} \mid y\right)$ and $V_{t}=\operatorname{var}\left(\alpha_{t} \mid y\right)$. To obtain these, we must run the state smoother which is a series of recursions that run in reverse order from the Kalman filter. These recursions are:

$$
\begin{aligned}
r_{t-1} & =Z_{t}^{\prime} F_{t}^{-1} v_{t}+L_{t}^{\prime} r_{t} \quad N_{t-1}=Z_{t}^{\prime} F_{t}^{-1} Z_{t}+L_{t}^{\prime} N_{t} L_{t} \\
\widehat{\alpha}_{t} & =a_{t}+P_{t} r_{t-1} \quad V_{t}=P_{t}-P_{t} N_{t-1} P_{t}
\end{aligned}
$$

for $t=T, \ldots, 1$.

We now have described the posterior for the unrestricted state space model (conditional on other model parameters) and, in particular, the posterior means and variances, $\widehat{\alpha}_{t}=E\left(\alpha_{t} \mid y\right)$ and $V_{t}=\operatorname{var}\left(\alpha_{t} \mid y\right)$. We now turn to the main focus of this paper: using the Savage-Dickey density ratio to compute dynamic posterior probabilities of restrictions on $\alpha_{t}$.

\section{Calculating Dynamic Posterior Probabilities of Restrictions using the SDDR}

The SDDR is a convenient way of calculating the Bayes factor comparing a restricted to an unrestricted model (call them $M_{R}$ and $M_{U}$ ). We begin with a general statement of the SDDR before applying it to the state space model. Suppose $M_{U}$, has a parameter vector $\theta=\left(\omega^{\prime}, \psi^{\prime}\right)^{\prime}$. The prior for this model is $p\left(\omega, \psi \mid M_{U}\right)$. The restricted version of the model, $M_{R}$, has $\omega=\omega_{0}$ where $\omega_{0}$ is a vector of constants. The parameters in $\psi$ are left unrestricted in each model. The prior for $M_{R}$ is $p\left(\psi \mid M_{R}\right)$. Suppose the priors in the two models satisfy: 


$$
p\left(\psi \mid \omega=\omega_{0}, M_{U}\right)=p\left(\psi \mid M_{R}\right),
$$

then the Bayes factor comparing $M_{R}$ to $M_{U}$ is:

$$
B F=\frac{p\left(\omega=\omega_{0} \mid y, M_{U}\right)}{p\left(\omega=\omega_{0} \mid M_{U}\right)}
$$

where $p\left(\omega=\omega_{0} \mid y, M_{U}\right)$ and $p\left(\omega=\omega_{0} \mid M_{U}\right)$ are the unrestricted posterior and prior for $\omega$ evaluated at the point $\omega_{0}$. Equation (5) is referred to as the SDDR. The proof that the SDDR is the Bayes factor is given in many places, including Verdinelli and Wasserman (1995). Note that (4) almost never restricts the form of the prior. For instance, if (as is commonly done), conditional on $\omega$, the same prior is used for parameters which are common to both models, then (4) is satisfied. In fact, it is an even weaker restriction than this, requiring only the same prior for common parameters to hold at one point $\left(\omega_{0}\right)$ in the parameter space. In this paper, we assume $(4)$ is satisfied. In the rare cases where this condition is not reasonable, Verdinelli and Wasserman (1995) provide a similar, but slightly more complicated expression for the Bayes factor and a simple extension of our methods is required.

Crucially, the SDDR involves only manipulations involving the posterior and prior for the unrestricted model which, in our case is the state space model described in the preceding section. Other approaches to Bayes factor calculation (e.g. calculating the marginal likelihood separately for the restricted and unrestricted models) would require estimation of the restricted model. For state space models with restrictions of the sort we consider (i.e. restrictions imposed at time $t$, but not necessarily at other times), we know of no available Bayesian methods for estimating the restricted model.

Here we derive the SDDR for restrictions on $\alpha_{t}$ of the form $A \alpha_{t}=\alpha^{*}$ where $A$ is a known $q \times m$ matrix and $\alpha^{*}$ is a known $q \times 1$ vector. Our derivations are greatly simplified due to the fact that $\alpha_{t}$ and, thus, $A \alpha_{t}$, have priors and posteriors which, conditional on the other model parameters, are Normal.

Using the SDDR, the Bayes factor comparing the restricted to the unrestricted model is:

$$
B F=\frac{p\left(A \alpha_{t}=\alpha^{*} \mid y\right)}{p\left(A \alpha_{t}=\alpha^{*}\right)},
$$


where the posterior and the prior in (6) are those for the unrestricted state space model described in the previous section.

We first discuss evaluating the posterior term in the numerator of (6). To make the notation compact, let $\phi=\left\{H_{t}, T_{t}, Q_{t}, a_{1}, P_{1}\right\}$ denote all the parameters (or pre-selected constants) in the state space model, other than the states themselves. Our results of the previous section tell us that, even though $p\left(\alpha_{t}=\alpha^{*} \mid y\right)$ does not have a convenient analytical form, $p\left(\alpha_{t}=\alpha^{*} \mid y, \phi\right)$ is Normal. To be precise,

$$
p\left(\alpha_{t} \mid y, \phi\right)=(2 \pi)^{-m / 2}\left|V_{t}\right|^{-1 / 2} \exp \left\{-\frac{1}{2}\left(\alpha_{t}-\widehat{\alpha}_{t}\right)^{\prime} V_{t}^{-1}\left(\alpha_{t}-\widehat{\alpha}_{t}\right)\right\}
$$

Using standard results for the multivariate Normal distribution, we have

$$
p\left(A \alpha_{t} \mid y, \phi\right)=(2 \pi)^{-m / 2}\left|A V_{t} A^{\prime}\right|^{-1 / 2} \exp \left\{-\frac{1}{2}\left(A \alpha_{t}-A \widehat{\alpha}_{t}\right)^{\prime}\left(A V_{t} A^{\prime}\right)^{-1}\left(A \alpha_{t}-A \widehat{\alpha}_{t}\right)\right\},
$$

and, thus,

$$
p\left(A \alpha_{t}=\alpha^{*} \mid y, \phi\right)=(2 \pi)^{-m / 2}\left|A V_{t} A^{\prime}\right|^{-1 / 2} \exp \left\{-\frac{1}{2}\left(\alpha^{*}-A \widehat{\alpha}_{t}\right)^{\prime}\left(A V_{t} A^{\prime}\right)^{-1}\left(\alpha^{*}-A \widehat{\alpha}_{t}\right)\right\} .
$$

Given output from our MCMC algorithm $\left(\phi^{(r)}\right.$ for $\left.r=1, \ldots, R\right)$, we can approximate the numerator of $(6)$ by:

$$
\widehat{p}\left(A \alpha_{t}=\alpha^{*} \mid y\right)=\frac{1}{R} \Sigma_{r=1}^{R} p\left(A \alpha_{t}=\alpha^{*} \mid y, \phi^{(r)}\right)
$$

using (8). As usual in MCMC algorithms, $R$ can be chosen to ensure any desired accuracy of approximation and MCMC diagnostics can be used to monitor convergence.

We now turn to the prior term in the denominator of (6). We adopt a similar strategy as with the numerator, except using prior simulator output. That is, $p\left(\alpha_{t}=\alpha^{*}\right)$ does not have a convenient analytical form, but $p\left(\alpha_{t}=\alpha^{*} \mid \phi\right)$ is Normal. Thus, if we have output from a prior simulator $\left(\phi^{(s)}\right.$ for $\left.s=1, \ldots, S\right)$, we can approximate the denominator of (6) by:

$$
\widehat{p}\left(A \alpha_{t}=\alpha^{*}\right)=\frac{1}{S} \Sigma_{s=1}^{S} p\left(A \alpha_{t}=\alpha^{*} \mid \phi^{(s)}\right) .
$$

To derive $p\left(A \alpha_{t}=\alpha^{*} \mid \phi^{(s)}\right)$, we use (2) and (3). Firstly, we rewrite (3) as $\alpha_{1}=a_{1}+P_{1}^{1 / 2} z$ where $z \sim N\left(0, I_{m}\right)$. 
The expressions in (2) and (3) define the hierarchical prior for the states. Using these, we obtain

$$
\begin{aligned}
\alpha_{t} & =\Pi_{i=1}^{t-1} T_{i} \alpha_{1}+\sum_{j=1}^{t-1} \Pi_{i=j+1}^{t-1} T_{i} \eta_{j} \\
& =\Pi_{i=1}^{t-1} T_{i} a_{1}+\Pi_{i=1}^{t-1} T_{i} P_{1}^{1 / 2} z+\Sigma_{j=1}^{t-1} \Pi_{i=j+1}^{t-1} T_{i} \eta_{j}
\end{aligned}
$$

Since $\alpha_{t}$ is a linear function of Normally distributed independent random variables, $\alpha_{t}$ is Normally distributed with prior mean

$$
\underline{\alpha}_{t}=E\left(\alpha_{t}\right)=\Pi_{i=1}^{t-1} T_{i} a_{1}
$$

and prior variance

$$
\operatorname{var}\left(\alpha_{t}\right)=\underline{V}_{t}=\Sigma_{j=1}^{t-1} \Pi_{i=j+1}^{t} T_{i} Q_{j}\left(\Pi_{i=j+1}^{t} T_{i}\right)^{\prime}+\Pi_{i=1}^{t-1} T_{i} P_{1}\left(\Pi_{i=1}^{t-1} T_{i}\right)^{\prime}
$$

Thus, the prior (conditional on $\phi$ ) can be written as:

$$
p\left(\alpha_{t} \mid \phi\right)=(2 \pi)^{-m / 2}\left|\underline{V}_{t}\right|^{-1 / 2} \exp \left\{-\frac{1}{2}\left(\alpha_{t}-\underline{\alpha}_{t}\right)^{\prime} \underline{V}_{t}^{-1}\left(\alpha_{t}-\underline{\alpha}_{t}\right)\right\} .
$$

Thus, the necessary term in (9) is:

$$
p\left(A \alpha_{t}=\alpha^{*} \mid \phi\right)=(2 \pi)^{-m / 2}\left|A \underline{V}_{t} A^{\prime}\right|^{-1 / 2} \exp \left\{-\frac{1}{2}\left(\alpha^{*}-A \underline{\alpha}_{t}\right)^{\prime}\left(A \underline{V}_{t} A^{\prime}\right)^{-1}\left(\alpha^{*}-A \underline{\alpha}_{t}\right)\right\} .
$$

In summary, given output from a posterior simulator and (7) we can obtain the numerator of the SDDR given in (6). Output from a prior simulator and (11) can be used to approximate the denominator. Given the Bayes factor comparing the restricted and unrestricted models, the posterior probability of the restriction can be calculated.

\section{Extensions}

As previously discussed, it is simple to extend our methods to be recursive (i.e. to calculate probability of restrictions using data through time $t$ rather than full sample). In this section, we discuss other important cases which require only simple extensions of the methods introduced in the preceding section. These are restrictions

involving more than one point in time and nonlinear restrictions. For the sake of brevity, in this section we do not provide exact formulae for these, but note how they can be obtained in a straightforward fashion. 
So far we have focused on restrictions at a single point in time (i.e. $p\left(A \alpha_{t}=\alpha^{*} \mid y\right)$ ). Our derivations were based on the fact that the simulation smoother directly provides us with the mean and variance of the Normal marginal distribution $p\left(\alpha_{t} \mid y, \phi\right)$. However, there might be some cases where the reader is interested in restrictions involving more than one point in time. For instance, we might be interested in the restriction $\alpha_{t}=\alpha_{t+1}$ (i.e. that no change occurred between $t$ and $t+1$ ). Use of the SDDR for such a restriction would require knowledge of $p\left(\alpha_{t}, \alpha_{t+1} \mid y, \phi\right)$. But, as outlined below, this is Normal with means and variances that can be built up from Kalman filter and simulation smoother output. Hence, it is straightforward to extend our methods to calculate $p\left(\alpha_{t}=\alpha_{t+1} \mid y\right)$.

In general, many hypotheses of interest fall in the general framework $C \alpha=c$ where $C$ is a known $T m \times T m$ matrix and $c$ a known $T m \times 1$ vector. In the previous section, we have focussed on questions of the form: "What is the probability that a restriction/theory holds at time $t$ ?". Restrictions of the form $C \alpha=c$ can be used to address questions like: "What is the probability that a restriction/theory holds at all time periods?" or "What is the probability that a restriction/theory holds through the first half of the sample?", etc. Also many questions of relevance to the structural break literature can be put in this format. For instance, if $C$ is the standard first-difference matrix and $c=0$ then the restrictions of interest are $\alpha_{1}=\alpha_{2}=. .=\alpha_{T}$ and the parameters are not time varying (although, as noted in the introduction, Bayes factor for this restriction can be calculated using existing methods such as those of Fruhwirth-Schnatter, 2004). By deleting the $\tau^{\text {th }}$ row from the first-difference matrix we can create a $C$ which allows for a single structural break at time $\tau$ (and, by calculating the probability of this restriction for all possible values of $\tau$ we can treat the timing of the break as unknown). These are only a few of the many examples of linear restrictions across time periods which the researcher may be interested in.

Durbin and Koopman (2001), chapter 4 and Carter and Kohn (1994), Lemma 2.1 are two of the many places which describe how the mean vector and covariance matrix of the Normal density $p(\alpha \mid y, \phi)$ can be obtained. In particular, we have seen in the previous section how the means and variances of $\alpha_{t}$ can be obtained, so we are only missing the covariances. But output from the Kalman filter and state smoother algorithm provide us with the covariances between $\alpha_{t}$ and $\alpha_{j}$ :

$$
\operatorname{Cov}\left(\alpha_{t}, \alpha_{j} \mid y, \phi\right)=P_{t} L_{t}^{\prime} L_{t+1}^{\prime} \ldots L_{j-1}^{\prime}\left(I-N_{j-1} P_{j}\right)
$$


Thus, the Normal density $p(\alpha \mid y, \phi)$ can easily be pinned down. The means and covariance matrix of the Normal density $p(C \alpha \mid y, \phi)$ can be obtained and used in an analogous fashion to (8) to obtain the numerator of the SDDR. The denominator can be derived using a prior simulator as described in the preceding section. In short, Bayes factors for linear restrictions involving any linear combination of the elements of $\alpha$ can be obtained in a straightforward manner.

Dealing with nonlinear restrictions is only slightly more difficult. Suppose we are interested in calculating the Bayes factor comparing a restricted to an unrestricted model where the restricted model imposes $g\left(\alpha_{t}\right)=\alpha^{*}$ where $g($.$) is a vector of invertible functions. The Bayes factor would be$

$$
B F=\frac{p\left(g\left(\alpha_{t}\right)=\alpha^{*} \mid y\right)}{p\left(g\left(\alpha_{t}\right)=\alpha^{*}\right)} .
$$

To evaluate $B F$ using the methods of the preceding section would require the same prior and posterior simulator output as before plus the ability fo evaluate $p\left(g\left(\alpha_{t}\right) \mid y, \phi\right)$ and $p\left(g\left(\alpha_{t}\right) \mid \phi\right)$ at the restrictions. But these latter densities can be obtained using the change-of-variable theorem (i.e. combining the Normal form for $p\left(\alpha_{t} \mid y, \phi\right)$ and $p\left(\alpha_{t} \mid \phi\right)$ with the Jacobian of the transformation from the relationship $\left.\alpha_{t}=g^{-1}\left(\alpha^{*}\right)\right)$.

\section{Dynamic Evidence on the Phillips Curve}

There has been a resurgence of interest in the Phillips curve. Influential examples of this work include King and Watson (1994), Staiger, Stock and Watson (1997) and Sargent, Williams and Zha (2006). Of particular importance in these studies are the questions of whether there is a trade-off between unemployment and inflation and, if not, what is the non-accelerating inflation rate of unemployment (NAIRU). Sargent, Williams and Zha (2006) argue that, through the 1970s, US monetary authorities observed evidence that suggested there was a trade-off between unemployment and inflation. In the following decade this trade-off vanished and so the monetary authorities cut inflation as they expected no cost in terms of unemployment. Thus, we have a restriction of policy interest (i.e. that the long run Phillips curve is vertical) and other authors have presented evidence that the support for this restriction is changing over time (although this evidence involves looking at point estimates and credible intervals for functions of the time-varying parameters). This is an ideal setup for 
using our new approach to calculating the dynamic posterior probability of this restriction. ${ }^{1}$

Staiger, Stock and Watson (1997) use a time varying model with a vertical Phillips curve imposed at all points in time. This specification coincides with the expectations augmented Phillips curve and permits estimation of the NAIRU. Since they use a time varying model, they are able to report time varying estimates of the NAIRU. With this model, there exist points in the parameter space at which the NAIRU is not identified. This provides us with a second restriction for which we can calculate dynamic posterior probabilities.

We use a state space model of the same general form as Staiger, Stock and Watson (1997), although we do not always impose a vertical long run Phillips curve. We use data from 1953Q1 through 2006Q2 on the unemployment rate (seasonally adjusted civilian unemployment rate, all workers over age 16) and inflation rate (the annual percentage change in a chain-weighted GDP price index). ${ }^{2}$

If there were a trade-off between inflation and unemployment, we would expect peaks in unemployment to coincide or lead troughs in inflation. Figure (1) shows the actual behavior of these series. Peaks in unemployment seem to be followed by troughs in inflation. However, the strength of this relationship seems to vary over time. Through the 1970s, the peaks in unemployment appear almost coincident with peaks in inflation. It is likely that the relationship between the level of the two series has changed over time. At least it appears that there is not a consistently negative relationship between the two. When one looks at the relationship between the change in inflation and the level of unemployment in Figure (2), however, there does seem to be a negative relationship. This would be the case if the long run Phillips curve were vertical.

As discussed in King and Watson (1994) and in Sargent, Williams and Zha (2006), there are different ways of specifying the relationship between unemployment $\left(u_{t}\right)$ and inflation $\left(\pi_{t}\right)$. We use two specifications. In the first of these (which does not impose the vertical Phillips curve), the inflation rate $\left(\pi_{t}\right)$ is the dependent variable and, thus, in terms of our notation for the state space model: $y_{t}=\pi_{t}$. In the second (which does impose the vertical Phillips curve and, thus, allows for estimation of the NAIRU), the change in inflation is the dependent variable $\left(y_{t}=\Delta \pi_{t}\right)$. In the first specification the explanatory variables are $Z_{t}=\left(1, \pi_{t-1}, \pi_{t-2}, u_{t}, u_{t-1}, u_{t-2}\right)$. In the second $Z_{t}=\left(1,-\Delta \pi_{t-1}, u_{t}, u_{t-1}, u_{t-2}\right)$. Note that we are always using two lags of both variables, the

\footnotetext{
${ }^{1}$ It might seem a little incongruous that a long run relation might hold at one point in time and not another. It is probably more informative to think of the long run relation as an equilibrium towards which the model attracts the variables at that point in time.

${ }^{2}$ The data were obtained from the Federal Reserve Bank of St. Louis website, http://research.stlouisfed.org/fred2/.
} 


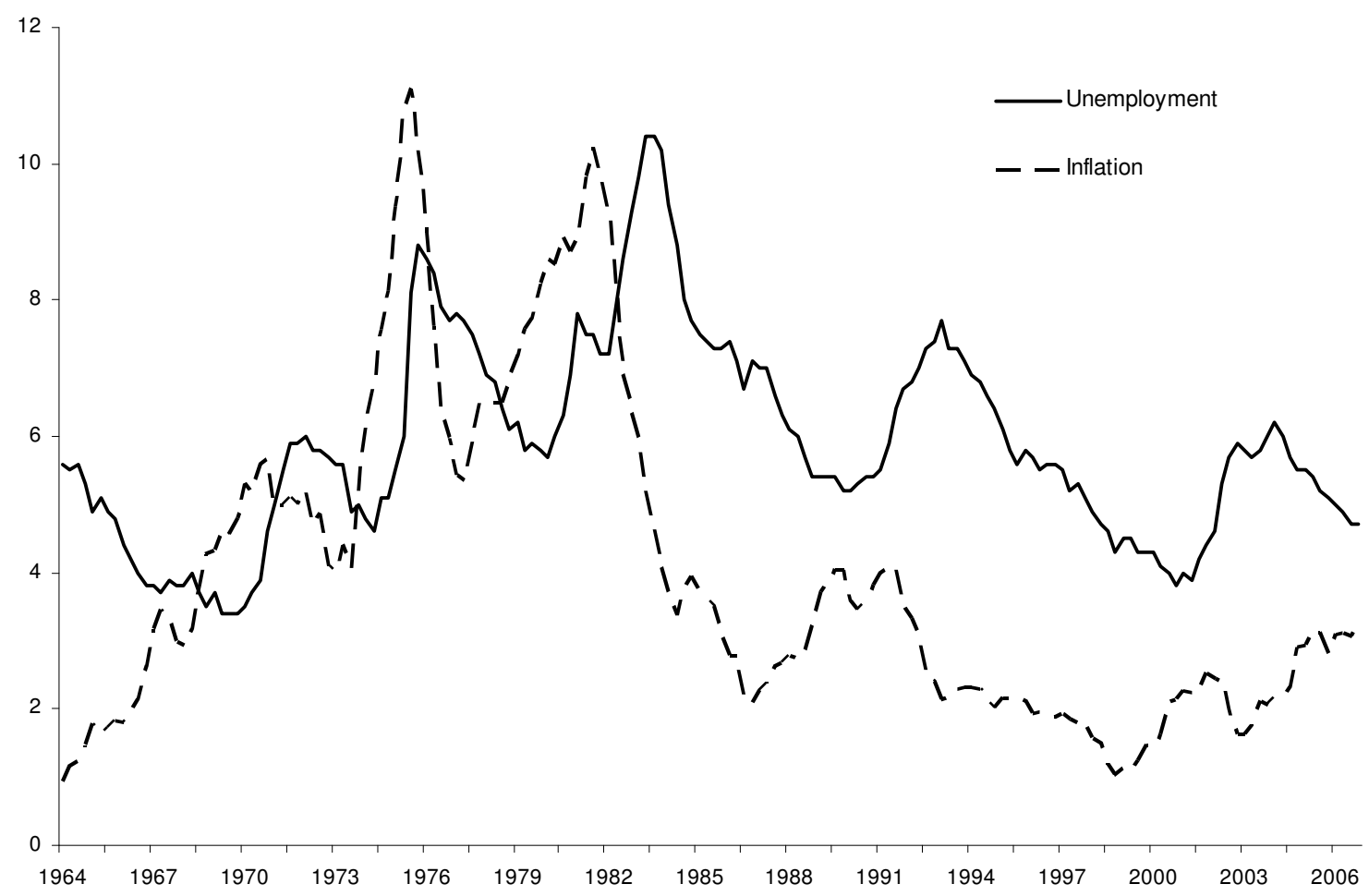

Figure 1: Inflation and unemployment levels.

same choice as Staiger, Stock and Watson (1997) and Sargent, Williams and Zha (2006).

To further explain these two specifications, ignore for now the time variation in parameters. Our first specification is an unrestricted one where inflation depends on lags of itself, current unemployment and lags of unemployment:

$$
\pi_{t}=\beta_{1}+\beta_{2} \pi_{t-1}+\beta_{3} \pi_{t-2}+\beta_{4} u_{t}+\beta_{5} u_{t-1}+\beta_{6} u_{t-2}+\varepsilon_{t} .
$$

A vertical long run Phillips curve is implied by the restriction that the coefficients on the lags of inflation sum to one: $\beta_{2}+\beta_{3}=1$. This is the first restriction of interest.

If we impose this restriction, we obtain:

$$
\Delta \pi_{t}=\beta_{1}-\beta_{3} \Delta \pi_{t-1}+\beta_{4} u_{t}+\beta_{5} u_{t-1}+\beta_{6} u_{t-2}+\varepsilon_{t} .
$$

Model (13) is the second specification we use in our empirical work. To see how it relates to the issue of the 


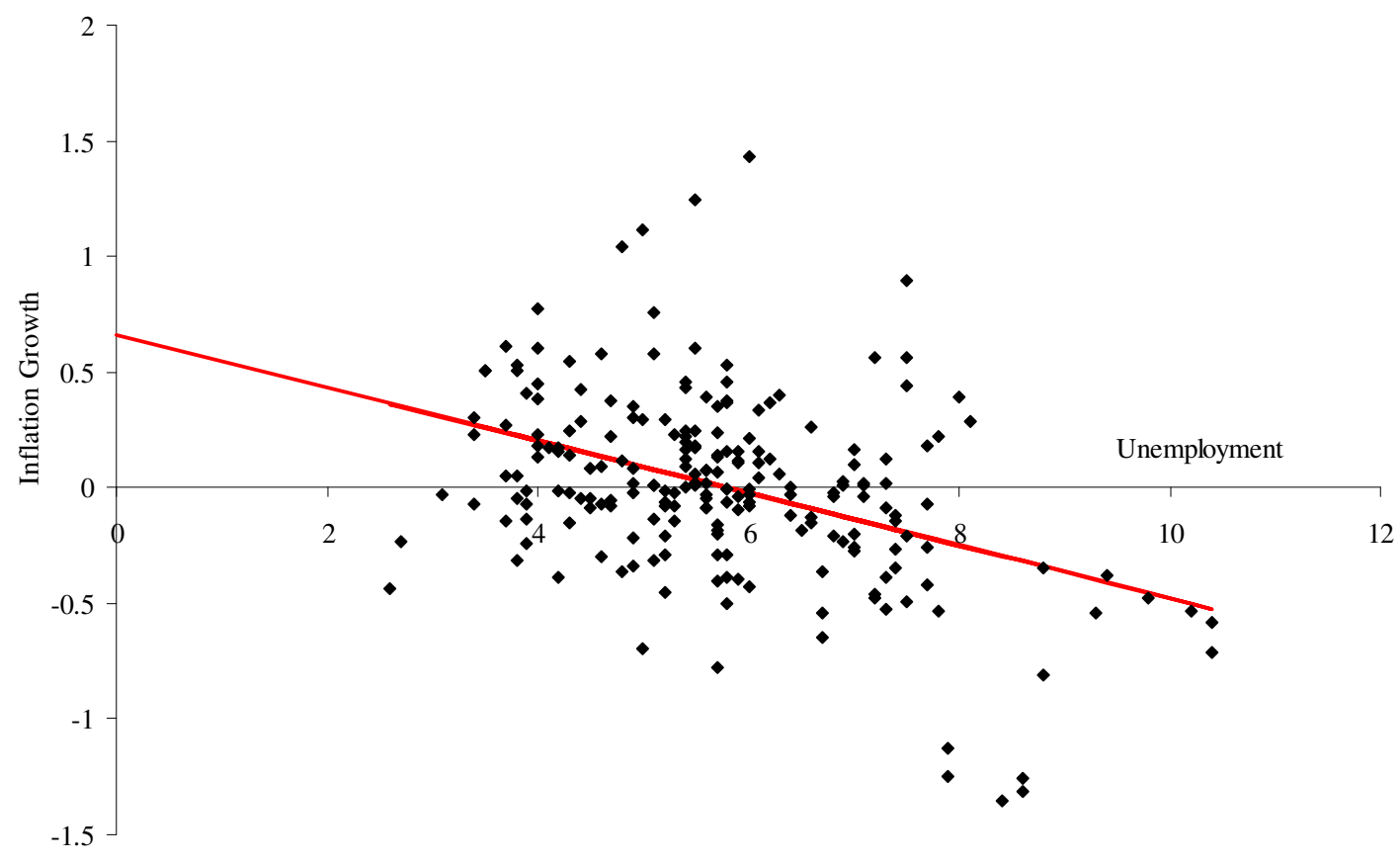

Figure 2: The change in inflation and the level of unemployment.

identification of NAIRU, we can rewrite it as:

$$
\Delta \pi_{t}=-\beta_{3} \Delta \pi_{t-1}+\beta_{4}\left(u_{t}-\underline{u}\right)+\beta_{5}\left(u_{t-1}-\underline{u}\right)+\beta_{6}\left(u_{t-2}-\underline{u}\right)+\varepsilon_{t},
$$

where $\underline{u}$ is the NAIRU. That is, (14) embeds the idea that it is deviations of unemployment from its natural rate which triggers inflation. The relationship between the coefficients in (13) and (14) is $\beta_{1}=-\underline{u}\left(\beta_{4}+\beta_{5}+\beta_{6}\right)$. From this, it can be seen that the NAIRU can be estimated from (13) provided $\beta_{4}+\beta_{5}+\beta_{6} \neq 0$. The NAIRU is not identified if $\beta_{4}+\beta_{5}+\beta_{6}=0$ which is our second restriction of interest.

In terms of the notation used for our state space model, we extend (12) to allow for time variation in coefficients by putting $t$ subscripts on the coefficients and letting: $\alpha_{t}=\left(\beta_{1, t}, \beta_{2, t}, \beta_{3, t}, \beta_{4, t}, \beta_{5, t}, \beta_{6, t}\right)^{\prime}$ and calculate the dynamic posterior probabilities that $\beta_{2, t}+\beta_{3, t}=1$. We extend (13) to allow for time variation in coefficients by setting: $\alpha_{t}=\left(\beta_{1, t}, \beta_{3, t}, \beta_{4, t}, \beta_{5, t}, \beta_{6, t}\right)^{\prime}$ and calculate the dynamic posterior probabilities that 
$\beta_{4, t}+\beta_{5, t}+\beta_{6, t}=0$. Since these are both linear restrictions on the states, our SDDR approach is directly applicable.

Before presenting empirical results, we must extend our model in a manner that is empirically important (but not of great relevance for either the economic or econometric theory discussed in this paper). It is well documented that the variance of inflation has changed markedly over time and, thus, stochastic volatility must be added to the model. We do this in a standard way and provide exact details in the appendix. In terms of the state space model in (1) and (2), the stochastic volatility assumption specifies $H_{t}$. We further assume $T_{t}=I$ and $Q_{t}=Q$. Details on priors and posterior simulation for the unknown parameters are given in the appendix. Suffice it to note here that we use a training sample prior of the sort commonly-used by empirical macroeconomist (see, e.g., Cogley and Sargent, 2001, 2005, Koop, Leon-Gonzalez and Strachan, 2007 and Primiceri, 2005). The general pattern of stochastic volatility that we find resembles that found in earlier studies using TVP models that allow both regression coefficients and error variances to vary (e.g., Primiceri, 2005, and Koop, Leon-Gonzalez and Strachan, 2007). There is a clear increase in volatility around the mid 1970s and another increase around 1980.

We now turn to our first restriction of interest: that the long run Phillips curve is vertical and, hence, that there exists a NAIRU. Figure (3) plots the Bayes factor in favor of this restriction. Kass and Raftery (1995) justifies and provides some useful rules of thumb for interpreting such Bayes factors. Note that our Bayes factor vary from 1.8 to 5.7. This is the region in which Kass and Raftery's rules of thumb suggest the evidence in support of the restriction is at worst 'Not worth more than a bare mention' and at best 'Positive'. The probability that this restriction holds tends to gradually fall from about $80 \%$ to $65 \%$ over the sample, with the exception of a peak of about $85 \%$ near the end of the 1970s. In short, we are finding some weak evidence in favor of the restriction that the long run Phillips curve is vertical, but this evidence is generally declining over time.

Since the evidence (although far from compelling) is always weakly in favor of the restriction that the long run Phillips curve is vertical, we proceed to our second model which is the TVP variant of (13). Remember that this allows for the calculation of the NAIRU provided that $\beta_{4, t}+\beta_{5, t}+\beta_{6, t} \neq 0$.

The Bayes factors in favor of $\beta_{4, t}+\beta_{5, t}+\beta_{6, t}=0$ are plotted in Figure (3). The dynamic Bayes factors in favor of the NAIRU not being identified are much more erratic (particularly in the period from the mid 
1970s through mid 1980s) than those we presented for the long run Phillips curve. Around the mid 1970s and again in the early 1980s there is evidence which approaches what Kass and Raftery's rules of thumb would say were 'Positive' and 'Strong' evidence against the restriction. The probability that the restriction holds falls as low as $28 \%$ in 1975 . However, at other periods, particularly after 1990 the evidence becomes 'Positive' for the restriction.

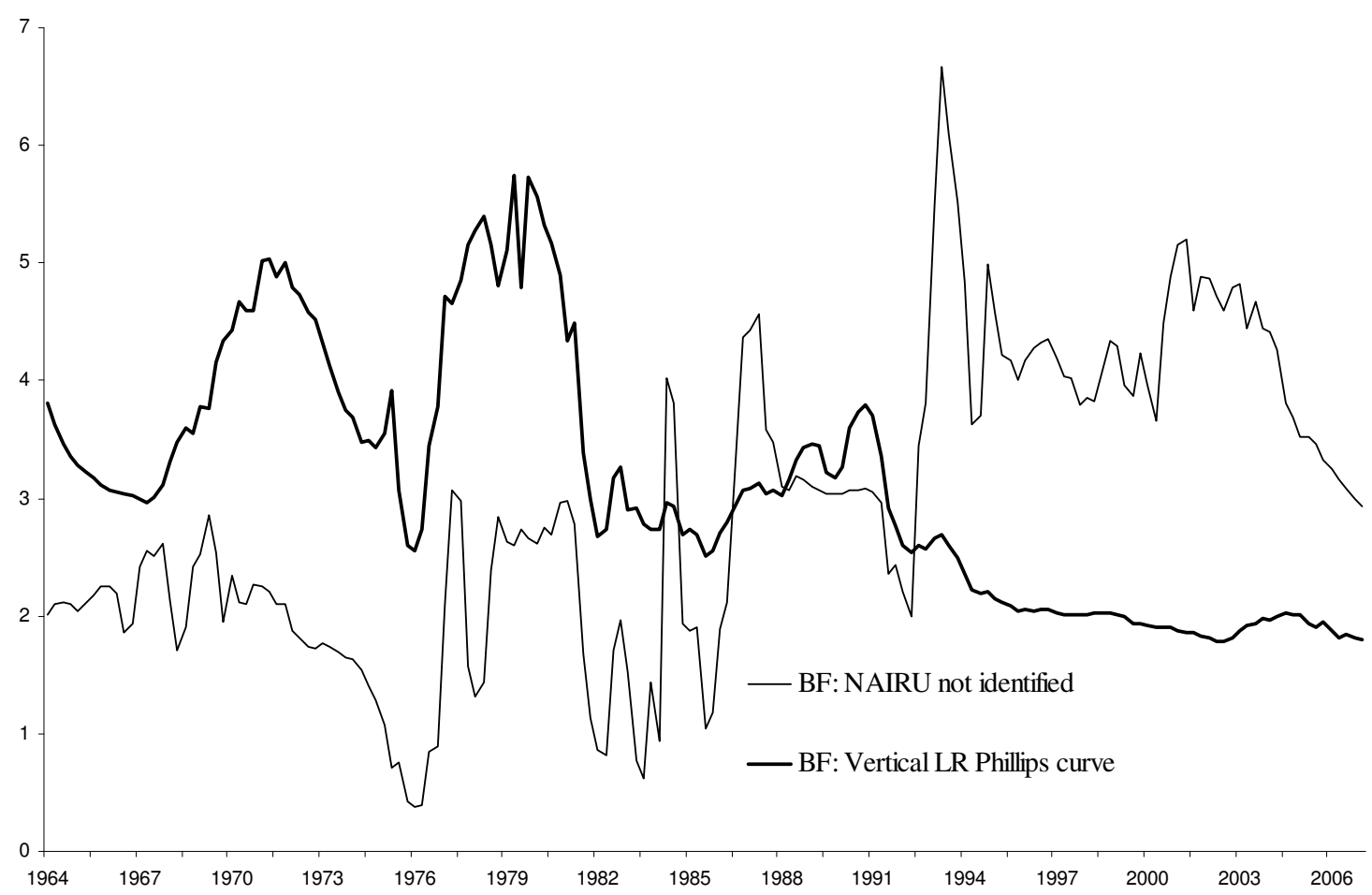

Figure 3: Bayes factors for $\beta_{2, t}+\beta_{3, t}=1$ (Vertical LR Phillips curve) and $\beta_{4, t}+\beta_{5, t}+\beta_{6, t}=0$ (NAIRU not identified).

The $16^{t h}, 50^{t h}$ and $84^{t h}$ percentiles of the posterior distribution of the NAIRU are plotted in Figure (4) along with the actual unemployment rate. ${ }^{3}$ We see a clear rise in the 1970s, followed by a gradual decline over the 1980s and 1990s. This same pattern was found by Staiger, Stock and Watson (1997).

\footnotetext{
${ }^{3}$ The point where the NAIRU is non-identified has zero mass and the posterior exists.
} 


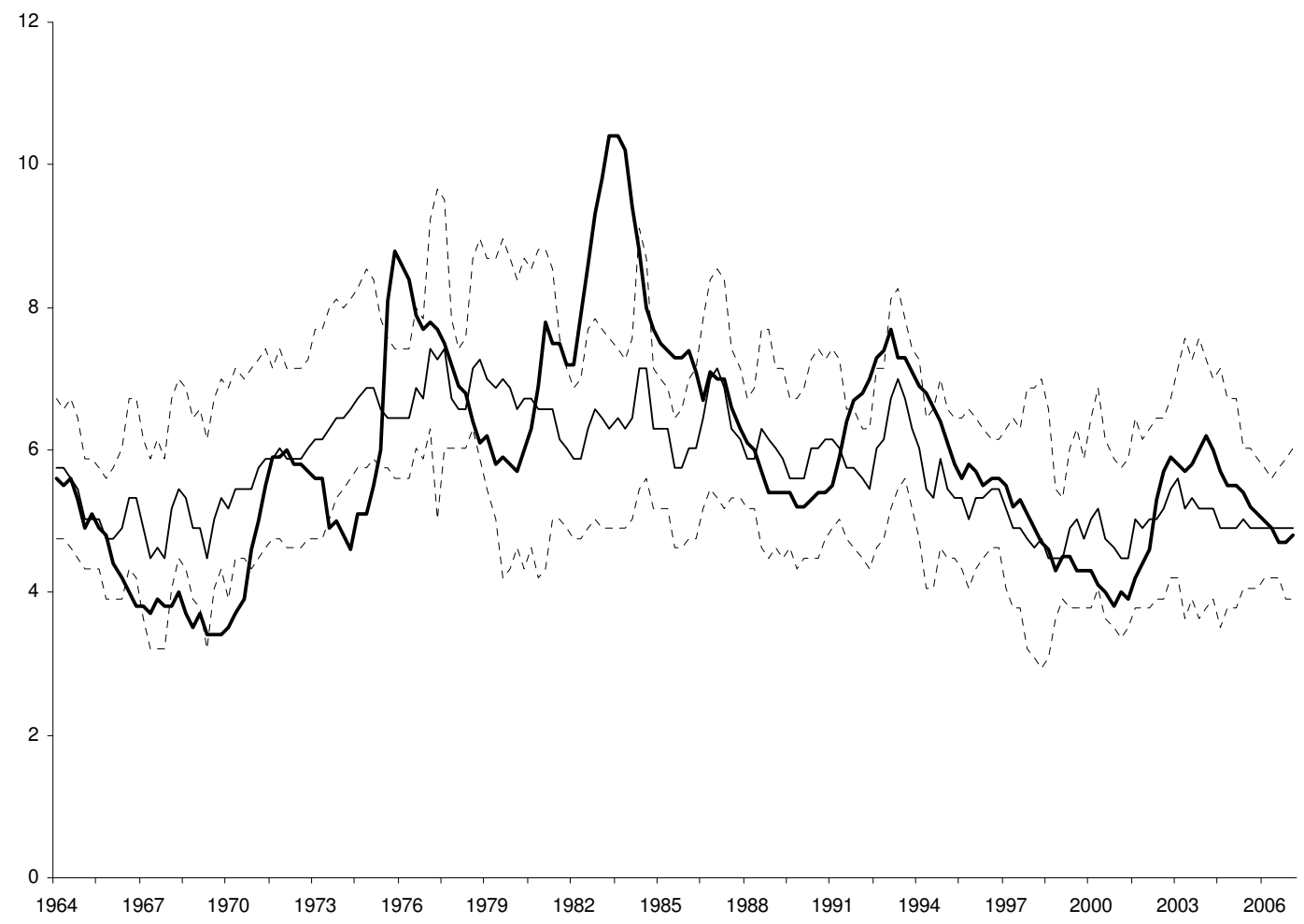

Figure 4: This figure plots the median (thin black line) and 16th and 84th percentiles (thin dashed line) of the posterior distribution for NAIRU. Also plotted is the actual unemployment rate (thick unbroken line).

\section{Conclusions}

In this paper, we have developed methods for calculating the dynamic posterior probability of restrictions on states in the state space model. This is of interest to the empirical macroeconomist since TVP-VAR and other time-varying parameter models are state space models and restrictions on the states are often suggested by economic theory. Our method for calculating the dynamic posterior probabilities are based is the Savage-Dickey density ratio which can be easily calculated using the output from standard MCMC algorithms for state space models.

We include an empirical application involving the unemployment and inflation rates. Our methods are used to calculate the probability that the long-run Phillips curve is vertical at each point in time. The probability that 
this restriction holds tends to be fairly high, but varies slightly over time. We also calculate the probability that another restriction, that the NAIRU is not identified, holds. There is slightly less evidence for this restriction, but the probability that it holds does fluctuate over time. 


\section{References}

Carter, C. and Kohn, R., 1994, On Gibbs sampling for state space models, Biometrika, 81, 541-553.

Cogley, T. and Sargent, T., 2001, Evolving post-World War II inflation dynamics, NBER Macroeconomic Annual, 16, 331-373.

Cogley, T. and Sargent, T., 2005, Drifts and volatilities: Monetary policies and outcomes in the post WWII U.S, Review of Economic Dynamics, 8, 262-302.

Durbin, J. and Koopman, S., 2001, Time Series Analysis by State Space Methods (Oxford University Press, Oxford).

Durbin, J. and Koopman, S., 2002, A simple and efficient simulation smoother for state space time series analysis, Biometrika, 89, 603-616.

Fruhwirth-Schnatter, S., 2004, Estimating marginal likelihoods for mixture and Markov switching models using bridge sampling techniques, Econometrics Journal, 7, 143-167.

Kass, R. and Raftery, A., 1995, Bayes Factors, Journal of the American Statistical Association, 90, 773-795.

Kim, S., Shephard, N. and Chib, S., 1998, Stochastic volatility: likelihood inference and comparison with ARCH models, Review of Economic Studies, 65, 361-93.

King, R., Plosser, C., Stock, J. and Watson, M., 1991, Stochastic trends and economic fluctuations, The American Economic Review, 81, 819-840.

King, R. and Watson, M., 1994, The post-war U.S. Phillips curve: a revisionist econometric history, CarnegieRochester Conference Series on Public Policy, 41, 157-219.

Koop, G., 2003, Bayesian Econometrics (Wiley, Chichester).

Koop, G., Leon-Gonzalez, R. and Strachan R.W., 2007, On the evolution of monetary policy, working paper available at http://personal.strath.ac.uk/gary.koop/koop_leongonzalez_strachan_kls5.pdf.

Koop, G., Potter, S. and Strachan, R,.W., 2007, Re-examining the consumption-wealth relationship: The role of model uncertainty, Journal of Money, Credit and Banking, forthcoming.

Lettau, M. and Ludvigson, S., 2004, Understanding trend and cycle in asset values: Reevaluating the wealth effect on consumption, American Economic Review, 94, 276-299.

Primiceri, G., 2005, Time varying structural vector autoregressions and monetary policy, Review of Economic 
Studies, 72, 821-852.

Sargent, T., Williams, N. and Zha, T., 2006, Shocks and government beliefs: The rise and fall of American inflation, American Economic Review, 96, 1193-1224.

Staiger, D., Stock, J. and Watson, M., 1997, The NAIRU, unemployment and monetary policy, Journal of Economic Perspectives, 11, 33-49.

Verdinelli, I. and Wasserman, L., 1995, Computing Bayes Factors using a generalization of the Savage-Dickey density ratio, Journal of the American Statistical Association, 90, 614-618. 


\section{Appendix}

In our empirical section, we use a special case of the state space model with $T_{t}=I$ and, thus, no additional details need to be provided about $T_{t}$. In terms of the parameters in the state space model, this leaves $Q_{t}, H_{t}$, $a_{1}$ and $P_{1}$.

We use a training sample prior that is very similar to that used in Cogley and Sargent (2001, 2005), Primiceri (2005) and Koop, Leon-Gonzalez and Strachan (2007). The main difference is that we average over different training samples. To be specific, we use a training sample prior with $\tau_{0}$ quarters of data to choose many of the key prior hyperparameters. That is, we first run a time-invariant regression using the first $\tau_{0}$ observations to produce OLS estimates of the regression coefficients, $\widehat{\beta}$, and the error variance, $\widehat{\sigma}^{2}$. We also obtain OLS estimates of the covariance matrix of $\widehat{\beta}$ which we label $\widehat{V}_{\beta}$. We use these quantities as prior hyperparameters as described below. To make sure our inferences are robust to choice of training sample, we average our results over $\tau_{0}=10,11,12, \ldots, 30$.

For the initial conditions in our state equation, we use:

$$
\alpha_{1} \sim N\left(\widehat{\beta}, 4 \widehat{V}_{\beta}\right)
$$

which defines $a_{1}$ and $P_{1}$. We set $Q_{t}=Q$ and use a conditionally conjugate Wishart prior for $Q^{-1}$. That is,

$$
Q^{-1} \sim W\left(\underline{\nu}_{Q}, \underline{Q}^{-1}\right)
$$

and the relevant posterior conditional used in the MCMC algorithm is:

$$
Q^{-1} \mid D a t a \sim W\left(\bar{\nu}_{Q}, \bar{Q}^{-1}\right)
$$

where

$$
\bar{\nu}_{Q}=T+\underline{\nu}_{Q}
$$

and

$$
\bar{Q}^{-1}=\left[\underline{Q}+\sum_{t=1}^{T}\left(\alpha_{t+1}-\alpha_{t}\right)\left(\alpha_{t+1}-\alpha_{t}\right)^{\prime}\right]^{-1} .
$$


We set $\underline{\nu}_{Q}=40$ and $\underline{Q}=0.0001 \widehat{V}_{\alpha}$.

Finally, a standard stochastic volatility model is used to specify $H_{t}$. In particular, if $h_{t}=\ln \left(H_{t}\right)$ is the log-volatility then we use:

$$
h_{t+1}=h_{t}+e_{t},
$$

where $e_{t}$ is $N\left(0, \sigma_{h}^{2}\right)$ and independent over $t$ and of $\varepsilon_{t}$ and $\eta_{t}$. Conditional on $\alpha$ and the other model parameters, we can use any standard algorithm for posterior simulation of the log-volatilities. In our empirical work, we use the algorithm of Kim, Shephard and Chib (1998). As was done by Primiceri (2005), for the initial condition, we take as prior:

$$
\log \left(h_{1}\right) \sim N\left(\log \left(\widehat{\sigma}^{2}\right), I_{3}\right)
$$

Finally, we use a Gamma prior for $\frac{1}{\sigma_{h}^{2}}$ :

$$
\frac{1}{\sigma_{h}^{2}} \sim G\left(\underline{h}, \underline{\nu}_{h}\right)
$$

where $G\left(\underline{h}, \underline{\nu}_{h}\right)$ denotes the Gamma distribution with mean $\underline{h}$ and degrees of freedom $\underline{\nu}_{h}$. The posterior for $\frac{1}{\sigma_{h}^{2}}$ (conditional on the states) is also Gamma:

$$
\frac{1}{\sigma_{h}^{2}} \mid D a t a \sim G\left(\bar{h}, \bar{\nu}_{h}\right)
$$

where

$$
\bar{\nu}_{h}=T+\underline{\nu}_{h}
$$

and

$$
\bar{h}=\frac{\bar{\nu}_{h}}{\underline{\nu}_{h} \underline{h}^{-1}+\sum_{t=1}^{T}\left(h_{t+1}-h_{t}\right)^{2}} .
$$

We set $\underline{\nu}_{h}=4$ and $\underline{h}^{-1}=0.0001 I_{3}$. 